\title{
A SPHERICAL SURFACE MEASURE INEQUALITY FOR CONVEX SETS
}

\author{
CHARLeS FefFERman, MAX JODEIT, JR. AND Michael D. PERLMAN ${ }^{1}$
}

\begin{abstract}
Let the set $C$ in the Euclidean space of $n$ dimensions be closed, symmetric under reflection in the origin, and convex. The portion of the surface of the unit ball lying in $C$ is shown to decrease in (the uniform) surface measure when $C$ is replaced by $A C$, the image of $C$ under any linear transformation $A$ with norm no greater than one. Some cases of equality are discussed, and an application is given.
\end{abstract}

1. Introduction. We prove that the hypersurface area of the intersection of a convex symmetric set $C \subseteq E^{n}(C=-C)$ with the unit sphere $S$ decreases when we shrink $C$. Precisely, let $\mu$ be the uniform surface measure on $S$ and let $A: E^{n} \rightarrow E^{n}$ be a linear transformation of norm $\leqq 1$. (The norm of $A$, denoted $\|A\|$, is given by $\|A\|=\sup \{|A x|: x \in S\}$, and $|y|^{2}=\sum y_{i}^{2}$.)

THEOREM 1. If $C$ is closed, convex, and symmetric about 0 , then $\mu(A C \cap S) \leqq \mu(C \cap S)$.

A simple geometric argument proves Theorem 1 in the two-dimensional case. First let $C$ be an infinite strip $L$ meeting $S$ in $I \cup(-I)$, the union of a symmetric pair of arcs (see Figure 1). Since the width of $A L$ is no greater than that of $L, \mu(A L \cap S) \leqq \mu(L \cap S)$.

Next assume $C$ is a polygon. Then $C \cap S$ may be expressed as the union of a finite collection of symmetric pairs of arcs $I_{j},-I_{j}$. For each $j$ let $L_{j}$ be the symmetric strip such that $L_{j} \cap S=I_{j} \cup\left(-I_{j}\right)$ (see Figure 2). Since $\|A\| \leqq 1$, the part of $C$ contained in the open unit ball $B$ plays no role (that is, $A C \cap S=A(C \sim B) \cap S)$. The remainder of $C$ is contained in the union

Received by the editors May 18, 1971.

AMS 1970 subject classifications. Primary 52A20, 52A40, 62H10.

Key words and phrases. Symmetric convex sets, geometric inequality, hypersurface area, second derivative test, radial measure, symmetric distributions, scale parameter family.

${ }^{1}$ The first two named authors partially supported by NSF GP-23563. The third author partially supported by NSF GP-21074 and 25911. The second author also partially supported by a Sloan Foundation grant during preparation of this paper. 


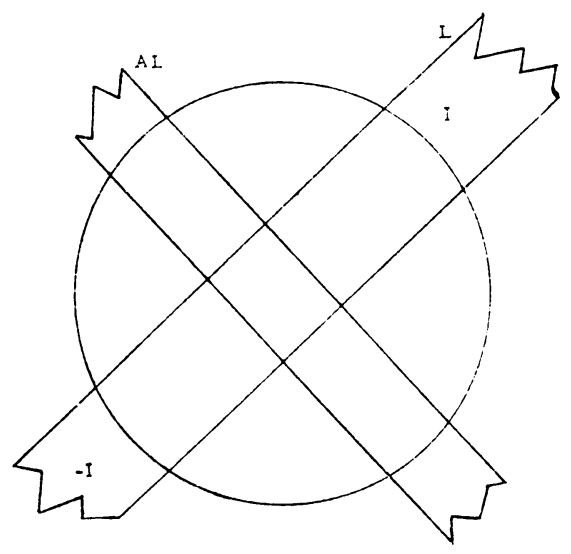

Figure 1

of the strips $L_{j}\left((C \sim B) \subseteq \bigcup L_{j}\right)$. Therefore, $A C \cap S \subseteq U\left(A L_{j} \cap S\right)$ so

$$
\begin{aligned}
\mu(A C \cap S) & \leqq \mu\left(\bigcup\left(A L_{j} \cap S\right)\right) \leqq \sum_{j} \mu\left(A L_{j} \cap S\right) \leqq \sum \mu\left(L_{j} \cap S\right) \\
& =\sum \mu\left(I_{j} \cup\left(-I_{j}\right)\right)=\mu(C \cap S)
\end{aligned}
$$

In the general case, a bounded $C$ is the intersection of a decreasing sequence of convex symmetric polygons $P_{n}$. Thus $\mu(A C \cap S) \leqq \mu\left(A P_{n} \cap S\right) \leqq$ $\mu\left(P_{n} \cap S\right) \rightarrow \mu(C \cap S)$ as $n \rightarrow \infty$. If $C$ is unbounded, $C$ must be a line, a strip or the whole plane.

In the case $n \geqq 3$ we could not find a geometric argument. Indeed, for $n=3$ the convex symmetric hull of a triangle lying outside $S$ offers difficulty. (See [2, Example (D), p. 407].)

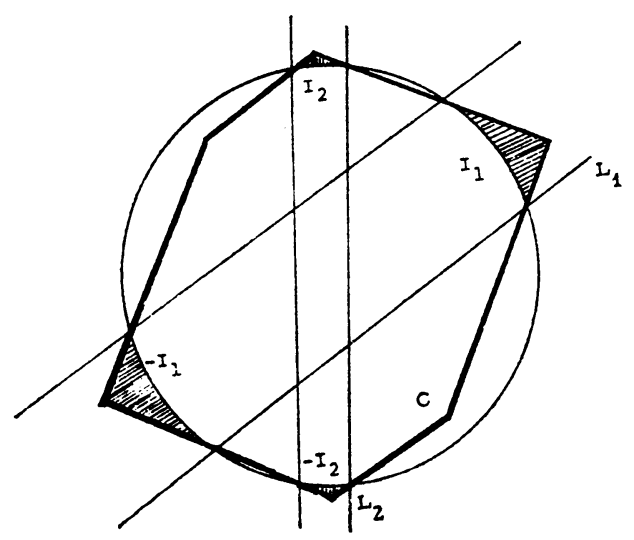

Figure 2 
Theorem 1 is false if symmetry is dropped, if "convex" is replaced by "star-shaped", or if $S$ is replaced by the surface of an ellipsoid.

The result is strengthened in Theorem 2 and the case of equality is discussed. An application to probability distributions is given in $\S 4$, extending results appearing in [1], [2], and [3].

We are grateful to U. C. Cramp, S. Das Gupta, and M. L. Eaton for stimulating discussions. We also extend thanks to Fred Flowers for drawing the diagrams.

2. Proof of Theorem 1. We make use of the following corollary of a theorem of Anderson [1] (see Sherman's interesting paper [4]). Let $f_{1}, f_{2} \geqq 0$ be even, bounded, and integrable on $E^{n}$, such that $\left\{x: f_{i}(x) \geqq \alpha\right\}$ is convex for each $\alpha$. Then for $x \in E^{n}, f_{1} * f_{2}(t x)$ is a nonincreasing function of $t>0$.

We can take $C$ compact. Since $C$ is a decreasing limit of closed convex symmetric polyhedra, we can take $C$ to be an arbitrary such polyhedron. Use orthogonal transformations to reduce $A$ to a matrix $D=$ $\operatorname{diag}\left(\lambda_{1}, \cdots, \lambda_{n}\right), 0 \leqq \lambda_{i} \leqq 1$. To show that $\mu(D C \cap S)$ is a nondecreasing function of each $\lambda_{i}$ it suffices to show that

$$
u(\lambda) \equiv \mu\left(D_{\lambda} C \cap S\right)=\int_{S} \chi_{C}\left(D_{\lambda}^{-1} x\right) d \mu(x)
$$

is a nondecreasing function of $\lambda(0<\lambda \leqq 1)$ where $D_{\lambda}=\operatorname{diag}(\lambda, 1, \cdots, 1)$, and $\chi_{C}$ is the indicator function of $C$.

For $\varepsilon>0$ let $f_{\varepsilon}$ be the $\left(C^{\infty}\right)$ approximate identity

$$
f_{\varepsilon}(x)=(2 \pi \varepsilon)^{-n / 2} \exp \left[-|x|^{2} / 2 \varepsilon\right]
$$

set $\varphi_{\varepsilon}=\chi_{C} * f_{\varepsilon}$ and

$$
u_{\varepsilon}(\hat{\lambda})=\int_{S} \varphi_{\varepsilon}\left(D_{\lambda}^{-1} x\right) d \mu(x) .
$$

Note that $\varphi_{\varepsilon}$ is $C^{\infty}$ and $\varphi_{\varepsilon}(x) \rightarrow \chi_{C}(x)$ as $\varepsilon \rightarrow 0$ (unless $x \in \partial C$; but $\mu(\partial C \cap S)=$ 0 as $C$ is a polyhedron) so $u_{\varepsilon}(\lambda) \rightarrow u(\lambda)$. Therefore it suffices to show that $u_{\varepsilon}^{\prime}(\lambda) \geqq 0$. We put $\psi(x)=\varphi_{\varepsilon}\left(D_{\lambda}^{-1} x\right)$ and write

$$
u_{\varepsilon}^{\prime}(\lambda)=-\lambda^{-1} \int_{S} x_{1} \frac{\partial \psi}{\partial x_{1}} d \mu(x) .
$$

Consider now $\chi_{B} * \psi$, where $B$ is the unit ball. It follows from the corollary to Anderson's theorem that $\chi_{B} * \psi$ has its maximum at the origin. Hence, with $z=(1,0, \cdots, 0)$,

$$
0 \geqq \frac{d^{2}}{d t^{2}}\left[\left(\chi_{B} * \psi\right)(t z)\right]_{t=0}=\left.\frac{\partial^{2}}{\partial x_{1}^{2}}\left(\chi_{B} * \psi\right)\right|_{x=0} .
$$


Write $x=\left(x_{1}, x^{\prime}\right)$ where $x^{\prime}=\left(x_{2}, \cdots, x_{n}\right) \in E^{n-1}$ and let $a\left(x^{\prime}\right)=\left(1-\left|x^{\prime}\right|^{2}\right)^{1 / 2}$. Then

$$
\begin{aligned}
0 & \geqq \int_{B} \frac{\partial^{2} \psi}{\partial x_{1}^{2}} d x \\
& =\int_{\left|x^{\prime}\right| \leqq 1}\left[\int_{-a\left(x^{\prime}\right)}^{a\left(x^{\prime}\right)} \frac{\partial^{2} \psi\left(x_{1}, x^{\prime}\right)}{\partial^{2} x_{1}} d x_{1}\right] d x^{\prime} \\
& =2 \int_{\left|x^{\prime}\right| \leqq 1} \frac{\partial \psi\left(a\left(x^{\prime}\right), x^{\prime}\right)}{\partial x_{1}} d x^{\prime} \\
& =\int_{S} x_{1} \frac{\partial \psi}{\partial x_{1}} d \mu(x)=-\lambda u_{\varepsilon}^{\prime}(\lambda) \text { Q.E.D. }
\end{aligned}
$$

3. A stronger result and some cases of equality. Theorem 1 implies that for a (not necessarily closed) convex symmetric set $C$,

$$
\mu(\bar{C} \cap S)=\mu\left(C^{0} \cap S\right)+\mu(\partial C \cap S) \geqq \mu(A \bar{C} \cap S) .
$$

In Theorem 2 we show that $\mu(\partial C \cap S)$ is not needed for nontrivial $A$ (even though $\mu\left(C^{0} \cap S\right)$ may be 0 ).

THEOREM 2. If $\|A\| \leqq 1$ but .4 is not orthogonal, then

$$
\mu(A \bar{C} \cap S) \leqq \mu\left(C^{0} \cap S\right) .
$$

Proof. Again we can take $A=D_{\lambda}=\operatorname{diag}(\lambda, 1, \cdots, 1)$ where now $0<\lambda<1$. For $\lambda<t<1$, Theorem 1 gives

$$
\mu\left(D_{\lambda} \bar{C} \cap S\right) \leqq \mu\left(D_{t} \bar{C} \cap S\right)=\int_{S} \chi_{i}(x) d \mu(x)
$$

where $\chi_{t}$ denotes the indicator function of $D_{t} \bar{C}$. We show that as $t \nearrow 1$, $\chi_{t \rightarrow \chi_{C 0} \cap S}$ almost everywhere in $S$. By symmetry restrict attention to the half-space $H=\left\{x \mid x_{1}>0\right\}$. There are four cases. If (i) $x \in C^{0} \cap H$ then $\chi_{t}(x) \rightarrow 1$ as $t \nearrow 1$. If (ii) $x \in \partial C \cap H$ but $D_{a} x \notin \bar{C}$ for $a>1$ (i.e., $x$ belongs to the "upper boundary" of $C$ ) then $\chi_{C^{0} \cap S}(x)=0=\chi_{t}(x)$ for $t<1$. Two cases remain: (iii) $x \in \partial C \cap H$ and $D_{1+\varepsilon} x \in \partial C$ for small $\varepsilon>0$ (i.e., $x \in H \cap L_{1}$, where $L_{1}$ is the "lateral boundary" of $C$ ) and (iv) $x \in \partial C \cap H$ and $D_{1+\varepsilon} x \in C^{0}$ for small $\varepsilon>0$ (i.e., $x \in H \cap L_{2}$, where $L_{2}$ is the "lower boundary" of $C$ ). In (iii) the projection of $L_{1}$ into $H_{0}=\left\{x \mid x_{1}=0\right\}$ lies in the boundary of the projection of $C$ into $H_{0}$, so $\mu\left(H \cap L_{1} \cap S\right)=0$. Finally, $\mu\left(H \cap L_{2} \cap S\right)=0$ by the following lemma, since $L_{2}$ is the graph of a convex function.

LEMMA. If $G \subset E^{n}$ is the graph of a nonnegative convex function then $\mu(G \cap S)=0$. 
Proof. Induction. The case $n=2$ is obvious. For $n \geqq 3$ first integrate over sections parallel to the "vertical" axis; each such integral is zero by induction. Q.E.D.

The case of equality in (1) has not been settled. In two dimensions it is easy to show from the geometric argument that strict inequality holds unless $A C \cap S=L C^{0} \cap S$ up to a set of $\mu$-measure 0 , for some orthogonal $L$. In the special case $\|A\|<1$, equality holds in (1) if and only if

$$
C^{0} \cap S=\varnothing \text { or } A \bar{C} \cap S=S .
$$

Sufficiency is clear. If (2) fails then $\mu\left(C^{0} \cap S\right)>0$ and $\mu(A \bar{C} \cap S)<\mu(S)$, so we can assume that $C^{0} \cap S \neq S$. We can also take $A=\operatorname{diag}\left(d_{1}, \cdots, d_{n}\right)=D$ with $0 \leqq d_{i}<1$; let $d=\max \left\{d_{i}\right\}<1$. By Theorem 2, $\mu(D \bar{C} \cap S) \leqq$ $\mu(d \bar{C} \cap S) \leqq \mu\left(C^{0} \cap S\right)$ so it suffices to show that $\mu(d \bar{C} \cap S)<\mu\left(C^{0} \cap S\right)$. Let $x \in d \bar{C} \cap S$. Then $y=d^{-1} x \in \bar{C}$ so $x \in[0, y) \subset C^{0}$. Thus $d \bar{C} \cap S$ (closed in $S$ ) is contained in $C^{0} \cap S$ (open in $S$ ). Since $C^{0} \cap S$ is a proper subset of $S$ this containment must be strict.

5. Extension to radial measures. Let $\nu$ be a radial Borel measure on $E^{n}$. That is, $\nu(E)=v(L E)$ for every Borel set $E$ and orthogonal transformation $L$. For any positive definite $\Sigma: E^{n} \rightarrow E^{n}$ define $\nu_{\Sigma}(E)=\nu\left(\Sigma^{-1 / 2} E\right)\left(\Sigma^{1 / 2}\right.$ can be any square root of $\Sigma$ since $v$ is radial). Theorem 2 easily gives the following result.

THEOREM 3. If $C$ is convex, symmetric, and if $\Sigma_{2}-\Sigma_{1}$ is positive semidefinite with $\Sigma_{1} \neq \Sigma_{2}$ then $v_{\Sigma_{2}}(\bar{C}) \leqq v_{\Sigma_{1}}\left(C^{0}\right)$.

If $v$ is a probability measure this result has the following interpretation. The family $\left\{v_{\Sigma}\right\}$ is a generalized scale parameter family of symmetric distributions. (If $v$ has finite second moments then the covariance matrix of $\nu_{\Sigma}$ is proportional to $\Sigma$.) Theorem 3 says that as the scale parameter (or covariance matrix) $\Sigma$ decreases, the distribution $\nu_{\Sigma}$ becomes more concentrated, or "peaked", about the origin in the sense of Sherman [4], i.e., the probability of every convex symmetric set is increased. This was proved for the Gaussian distribution by Anderson [1, Corollary 3, p. 173]. Also see Dudley, Feldman and LeCam, [2, (A), p. 406], and Gross [3, Lemma 5.2, p. 384]. The case of the uniform distribution on the unit ball is proved by Gross [3, Lemma 5.1, p. 383].

\section{REFERENCES}

1. T. W. Anderson, The integral of a symmetric unimodal function over a symmetric convex set and some probability inequalities, Proc. Amer. Math. Soc. 6 (1955), 170-176. MR 16, 1005.

2. R. M. Dudley, J. Feldman and L. LeCam, On seminorms and probabilities, and abstract Wiener spaces, Ann. of Math. (2) 93 (1971), 390-408. 
3. L. Gross, Measurable functions on Hilbert space, Trans. Amer. Math. Soc. 105 (1962), 372-390. MR 26 \#121.

4. S. Sherman, A theorem on convex sets with applications, Ann. Math. Statist. 26 (1955), 763-767. MR 17, 655.

Department of Mathematics, University of Chicago, Chicago, Illinois 60637

Current address (Michael D. Perlman): Department of Statistics, University of Minnesota, Minneapolis, Minnesota 55455 